\title{
Serum microRNA expression signatures as novel noninvasive biomarkers for prediction and prognosis of muscle-invasive bladder cancer
}

\author{
Xiumei Jiang ${ }^{1}$, Lutao Du ${ }^{1}$, Weili Duan ${ }^{1}$, Rui Wang ${ }^{1}$, Keqiang Yan², Lili Wang ${ }^{1}$, Juan \\ $\mathbf{L i}^{1}$, Guixi Zheng ${ }^{1}$, Xin Zhang ${ }^{1}$, Yongmei Yang ${ }^{1}$, Chuanxin Wang ${ }^{1}$ \\ ${ }^{1}$ Department of Clinical Laboratory, Qilu Hospital, Shandong University, Jinan, 250012, Shandong Province, China \\ ${ }^{2}$ Department of Urology, Qilu Hospital, Shandong University, Jinan, 250012, Shandong Province, China \\ Correspondence to: Chuanxin Wang, e-mail: cxwang@sdu.edu.cn \\ Keywords: muscle-invasive bladder cancer, microRNA, prediction, prognosis, serum
}

Received: September 29, 2015

Accepted: April 16, 2016

Published: May 4, 2016

\section{ABSTRACT}

Noninvasive biomarkers for predicting the risk of muscle-invasive bladder cancer (MIBC) may expedite appropriate therapy and reduce morbidity and cost. Genome-wide miRNA analysis by Miseq sequencing followed by two phases of reverse transcription quantitative real-time PCR (RT-qPCR) assays were performed on serum from 207 MIBC patients, 285 nonmuscle-invasive bladder cancer (NMIBC) patients and 193 controls. A four-miRNA panel (miR-422a-3p, miR-486-3p, miR-103a-3p and miR-27a-3p) was developed for MIBC prediction with an area under the receiver operating characteristic curve (AUC) of 0.894 (95\% CI, 0.846-0.931) for training set. Prospective evaluation of the miRNA panel revealed an AUC of 0.880 ( $95 \% \mathrm{CI}, 0.834$ to 0.917$)$ in validation set, which was significantly higher than those of grade and urine cytology (both $p<0.05$ ). Moreover, Kaplan-Meier analysis showed that MIBC patients with low miR-486-3p and miR-103a-3p levels had worse overall survival ( $p=0.002$ and $p=0.034$, respectively). Cox analysis indicated miR-486-3p and miR-103a-3p were independently associated with overall survival of MIBC ( $p=0.042$ and $p=0.021$, respectively). In conclusion, serum miRNA signatures might have considerable clinical values in predicting and providing prognostic information for MIBC.

\section{INTRODUCTION}

Bladder cancer (BC) is one of the most prevalent malignancies worldwide, with approximately 74,000 new cases and 16,000 new deaths in 2015 in the United States [1]. At initial presentation, $75 \%$ of tumors do not invade the muscle wall, whereas $30 \%$ will recur with muscleinvasive disease during follow up [2]. The remaining 25\% of tumors present with muscle-invasive disease which features a high mortality rate [3]. Currently, standard test used to measure the local stage of $\mathrm{BC}$ is transurethral resection (TUR) [4]. This method could provide guide on treatment of non-muscle-invasive BC (NMIBC) and staging information for muscle-invasive $\mathrm{BC}$ (MIBC). However, this approach is invasive, uncomfortable and costly. Although urine cytology is widely used as noninvasive test for diagnosis of $\mathrm{BC}$, its value in discriminating $\mathrm{MIBC}$ from NMIBC is limited owing to low accuracy [5]. Therefore, novel and noninvasive biomarkers are urgently needed to improve current strategies for prediction of tumor stage.

MicroRNAs (miRNAs) are a subset of small noncoding RNAs (typically 19-25 nucleotides in length) that increase and/or decrease gene expression at the posttranscriptional level [6,7]. Different miRNAs could function as tumor suppressors or oncogenes, and their expression has been associated with genesis and invasion of cancer [8-11]. Previous studies demonstrated that numerous miRNAs exist stably in human serum and have potential roles in diagnosis, staging and prediction of outcomes in various types of cancer [12-17]. Recently, we have identified a serum miRNA profile (miR-152, miR148b-3p, miR-3187-3p, miR-15b-5p, miR-27a-3p and miR-30a-5p) for diagnosis of BC via Miseq sequencing followed by two phases of reverse transcription quantitative real-time PCR (RT-qPCR) assays [18]. However, unique serum miRNA signatures that could predict tumor stage of $\mathrm{BC}$ have not been identified. 
In the present study, we designed an investigation following the similar strategy to test the hypothesis that stage-specific miRNAs exist in serum and can be useful in predicting MIBC with the hope that such findings may guide therapeutic choice. Moreover, correlation between serum miRNAs and prognosis of MIBC was further assessed.

\section{RESULTS}

\section{High-throughput sequencing of serum miRNAs from MIBC patients, NMIBC patients and controls}

Among the 567 serum miRNAs that were scanned by Miseq sequencing ( $\geq 1$ copy), 234, 278 and 193 miRNAs were detectable ( $\geq 10$ copies) in MIBC group, NMIBC group and control group, respectively. Expression of a miRNA was considered altered in MIBC only if at least 50 copies were detected by Miseq sequencing together with significant deregulations larger than two-fold change in MIBC vs. Normal, NMIBC vs. Normal, and MIBC vs. NMIBC comparisons. Based on these criteria, 23 miRNAs were selected as differentially expressed (Supplementary Table S1). miR-152, miR-148b-3p, miR-3187-3p, miR-15b$5 p$, miR-27a-3p and miR-30a-5p were also selected because they had been shown to have diagnostic value for $\mathrm{BC}$ in our previous study [18]. Thus, 29 miRNAs were selected as candidates for further testing via RT-qPCR.

\section{Evaluation of miRNA expression by RT-qPCR}

The 29 candidate miRNAs were first tested with RTqPCR using an independent cohort of 40 MIBC patients, 40 NMIBC patients and 40 controls. Four miRNAs (miR-422a-3p, miR-486-3p, miR-103a-3p and miR-27a$3 p)$ showed differential expression levels in MIBC vs. Normal, NMIBC vs. Normal, and MIBC vs. NMIBC comparisons (all at $p<0.05$, Supplementary Figure $\mathrm{S} 1$ ). The expression profile of these four miRNAs was further evaluated by RT-qPCR on additional 71 MIBC patients, 71 NMIBC patients and 147 controls. The four miRNAs were significantly differently expression among MIBC, NMIBC and control groups (all at $p<0.05$, Figure 1). The corresponding AUCs of these four miRNAs were 0.767, $0.718,0.706$, and 0.641 , respectively (Figure 2).

The concentrations of the four miRNAs were further measured using the validation cohort consisted of 90 MIBC patients and 168 NMIBC patients. Alterations in miRNA expression pattern of validation set were consistent with those of training set (Table 1 and Supplementary Figure S2).

\section{Establishment of the predictive miRNA panel}

A stepwise logistic regression model to estimate the risk of being predicted with MIBC was applied on training data set. The predicted probability of being diagnosed with
MIBC from the logit model based on the four-miRNA panel, logit $(p=$ MIBC $)=-1.5992+(0.4938 \times$ miR-422a$3 p)+(0.4197 \times$ miR- $486-3 p)+(0.4168 \times$ miR-103a- $3 p)$ $+(0.2335 \times$ miR-27a-3p) was used to construct the ROC curve. The AUC of the four-miRNA panel was 0.894 (95\% confidence interval [CI], 0.846 to 0.931 ) and the optimal cut-off value was 0.0282 , providing a sensitivity of $90.99 \%$ and a specificity of $72.97 \%$ (Figure 3A). A threshold of 0.0282 was selected to ensure good predictive ability for MIBC.

\section{Validation of the predictive miRNA panel}

The parameters estimated from the training set were used in a blind fashion to predict the probability of being diagnosed with MIBC for the independent validation data set. Using the classification threshold score of $<0.0282$ derived above, 132 samples were identified as noninvasive and 126 samples were identified as invasive tumors. After unblinding 121 of the 168 noninvasive tumors [specificity, $70.06 \%(95 \% \mathrm{CI}, 79.1$ to 90.1$)]$ and 79 of the 90 invasive tumors [sensitivity, $90.00 \%(95 \% \mathrm{CI}, 81.9$ to 95.3$)$ ] were correctly identified resulting in an AUC of 0.880 (95\% CI, 0.834 to 0.917 , Figure $3 \mathrm{~B}$ ). Analysis of these classification results demonstrated that accuracy for invasive tumors trended upwards the higher cancer stage (Table 2).

Although low-grade BCs are rarely muscle-invasive, high-grade tumors can be either muscle-invasive or non-muscle-invasive. We thus evaluated tumor grade in classifying muscle-invasive from non-muscle-invasive tumors and ROC analysis revealed an AUC of 0.779 (95\% CI, 0.724 to 0.828 ) (Figure 3C). The predictive performance of urine cytology in distinguishing MIBC from NMIBC was further evaluated on the validation set, and ROC analysis revealed an AUC of 0.602 (95\% CI, 0.539 to 0.662 ) (Figure 3D). The AUC of the four-miRNA panel for MIBC was markedly higher than those of tumor grade and urine cytology (both $p<0.05$ ).

\section{Correlations between miRNA expression levels and patient survival}

In the validation phase, 18 of the 90 MIBC patients were lost to follow-up and survival analysis was performed on the remaining 72 MIBC patients. The median followup time was 56 (range 3-73) months. Kaplan-Meier survival analysis revealed that MIBC patients with low miR-486-3p and miR-103a-3p expression levels showed significantly reduced overall survival (OS) than those with high miR-486-3p and miR-103a-3p levels ( $p=0.002$ and $p=0.034$, respectively) (Figure 4). Univariate Cox proportional hazards regression model analysis revealed that OS significantly correlated with miR-486-3p level ( $p$ $=0.004)$, miR-103a-3p level $(p=0.039)$, tumor stage $(p$ $=0.005)$ and lymph node status $(p=0.031)$. Parameters significantly related to OS in the univariate analysis were put into the multivariate analysis to identify independent 
factors for prognoses. The results showed that miR-486$3 p$ level $(p=0.042)$, miR-103a-3p level $(p=0.021)$, tumor stage $(p=0.030)$ and lymph node status $(p=0.025)$ maintained their significance as independent prognostic factors for OS of MIBC (Table 3).

A

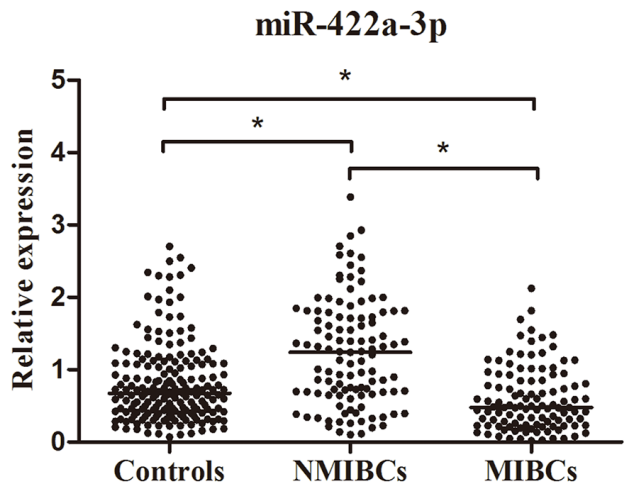

C

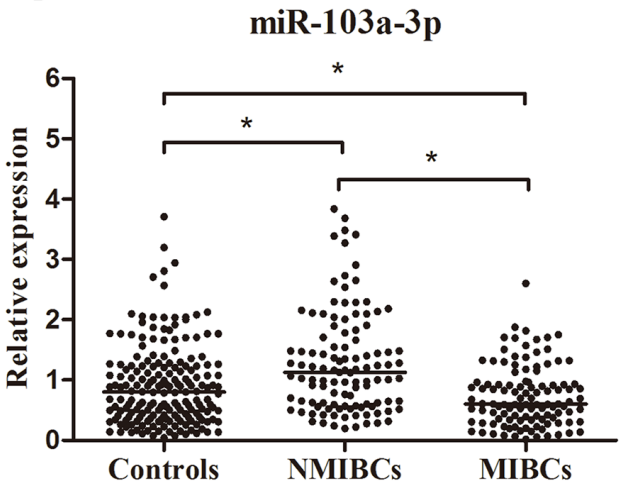

\section{DISCUSSION}

In $\mathrm{BC}$, identification of noninvasive and invasive phenotypes is vital to rational clinical management [19]. Previously, Schiffer et al. identified a panel of urinary
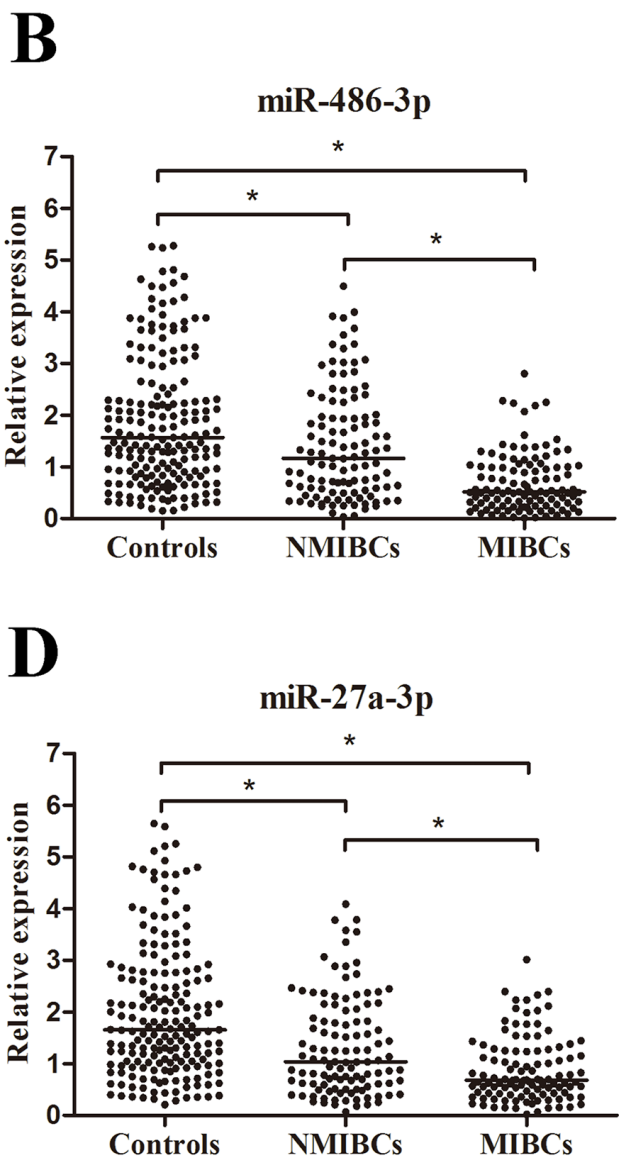

Figure 1: Concentrations of four miRNAs in MIBC patients $(n=111)$, NMIBC patients $(n=111)$ and control individuals $(\mathrm{n}=187)$ using RT-qPCR assay in training set A-D. * $p<0.05$.

A

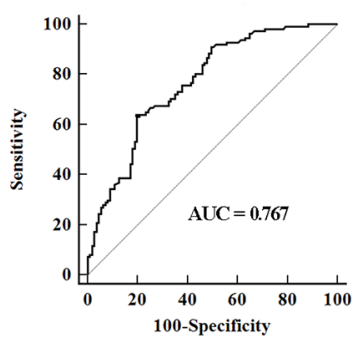

B

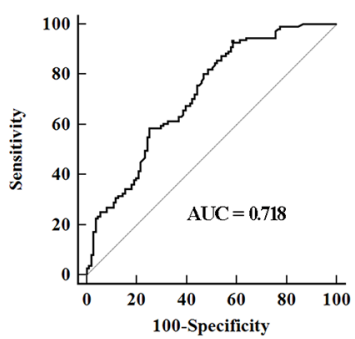

C

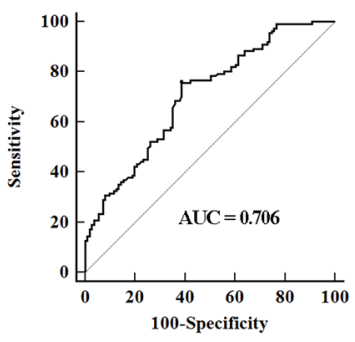

D

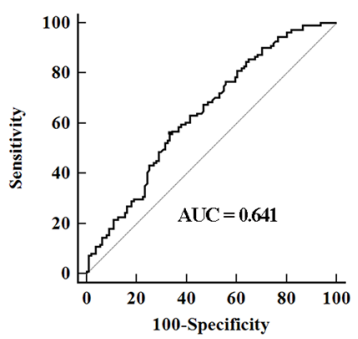

Figure 2: ROC curves analysis for detection of MIBC using A. miR-422a-3p, B. miR-486-3p, C. miR-103a-3p, D. miR27a-3p in MIBC patients $(n=111)$, NMIBC patients $(n=111)$ and control individuals $(n=187)$ in training set. 
peptides that seemed promising in predicting MIBC [5]. Yet, little was known about noninvasive miRNA biomarkers that can effectively accomplish this task. Catto et al. demonstrated that distinct miRNA alterations were associated with tumor progression of BC [20]. Wiklund et al. revealed coordinated epigenetic repression of the miR200 family and miR-205 in invasive BC and suggested significant correlation between miR-200c silencing and T1 tumor progression [21]. In addition, miR-10a-5p has been shown to be down-regulated in tissues of Ta representing cases with stage progression [22]. Although these studies provide interesting insights into tumor pathology of $\mathrm{BC}$, their effect in predicting tumor stage using serum still remains unclear. In the present study, our analysis revealed that miR-422a-3p, miR-486-3p, miR-103a-3p and $\mathrm{miR}-27 \mathrm{a}-3 \mathrm{p}$ were differently expressed in a MIBCspecific manner. Moreover, the four-miRNA signature demonstrated high accuracy for prediction of MIBC compared with tumor grade and traditional urine cytology. To our knowledge, this is the first study to characterize a serur miRNA expression signature for distinguishing muscle-invasive and non-muscle-invasive tumors of bladder by use of the genome-wide Miseq sequencing platform.

From a clinical perspective, the ability to predict muscle-invasive disease noninvasively could have important applications in urologic practice. Such biomarkers could offer pre-TUR information that may be useful for determination of the extent of TUR when MIBC is predicted. Under such conditions, when patients choose to have radical cystectomy, considering the benefit of neoadjuvant chemotherapy [23], such treatment can be implemented immediately. If patients want to undergo radiochemotherapy, complete TUR would be prepared.

Table 1: Expression of four miRNAs in serum in NMIBC patients and MIBC patients in training set and validation set [median (interquartile range)].

\begin{tabular}{lcccccc}
\hline miRNA & \multicolumn{3}{c}{ Training set } & \multicolumn{3}{c}{ Validation set } \\
\cline { 2 - 7 } & NMIBCs & MIBCs & $\boldsymbol{p}$ value & NMIBCs & MIBCs & $\boldsymbol{p}$ value \\
\hline miR-422a-3p & $1.24(0.67-1.79)$ & $0.48(0.23-0.85)$ & $<0.001$ & $1.08(0.65-1.53)$ & $0.53(0.33-0.79)$ & $<0.001$ \\
miR-486-3p & $1.61(0.54-2.01)$ & $0.52(0.25-1.01)$ & $<0.001$ & $1.04(0.70-1.74)$ & $0.55(0.36-0.92)$ & $<0.001$ \\
miR-103a-3p & $1.27(0.59-1.78)$ & $0.60(0.33-0.96)$ & $<0.001$ & $0.97(0.69-1.53)$ & $0.65(0.40-0.96)$ & $<0.001$ \\
miR-27a-3p & $1.04(0.59-2.03)$ & $0.68(0.42-1.25)$ & $<0.001$ & $1.04(0.67-1.81)$ & $0.71(0.47-1.23)$ & $<0.001$ \\
\hline
\end{tabular}

A

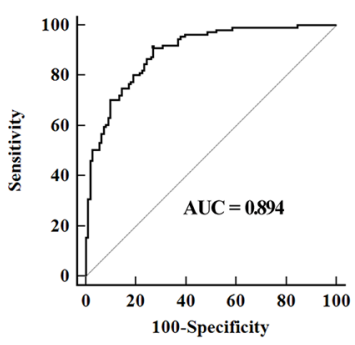

B

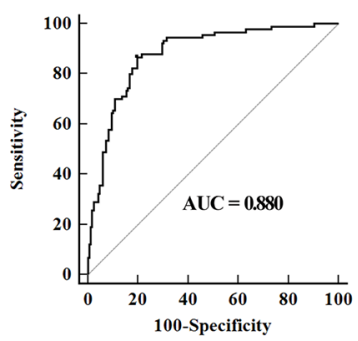

C

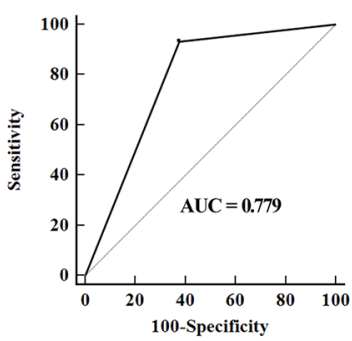

D

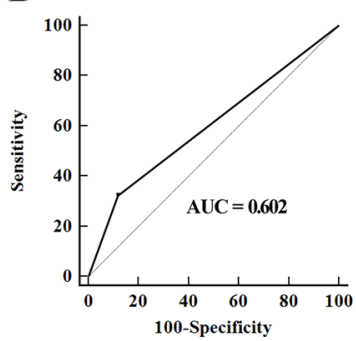

Figure 3: A-B. ROC curves for detection of MIBC using the four-miRNA panel in training set (A) and validation set (B); C. ROC curve analysis using grade for detection of $\mathrm{MIBC}$ in validation set; D. ROC curve analysis using urine cytology for prediction of MIBC in validation set.

Table 2: Tumor staging classification performance of miRNA panel in BC patients in validation set.

\begin{tabular}{lcc}
\hline Tumor stage & Specificity (95\% CI) & Sensitivity (95\% CI) \\
\hline Ta $(\mathrm{n}=55)$ & $61.82(47.7-74.6)$ & - \\
T1 $(\mathrm{n}=113)$ & $71.68(62.4-79.8)$ & - \\
T2 $(\mathrm{n}=28)$ & - & $78.57(59.0-91.7)$ \\
T3 $(\mathrm{n}=39)$ & - & $87.18(72.6-95.7)$ \\
T4 $(\mathrm{n}=23)$ & - & $95.65(78.0-99.3)$ \\
\hline
\end{tabular}


Furthermore, pathologists may have difficulty determining whether tumor has invaded the musclaris propria, which is crucial in choosing therapy. In such cases, these patients have to undergo a resection of the base of the tumor [24]. Here, a panel of serum miRNAs could provide relatively definite answer as to tumor stage evaluation, thus enabling a defined treatment pathway. Although numerous circulating miRNAs for detection of BC have been revealed [25-29], we provided the first evidence to support the hypothesis that serum miRNAs offer predictive value in distinguishing MIBC from NMIBC. Nevertheless, little was known about expression of serum miRNAs in recurrent MIBC and their predictive potential. As all patients with MIBC in this study were initially diagnosed, further studies are needed to validate whether

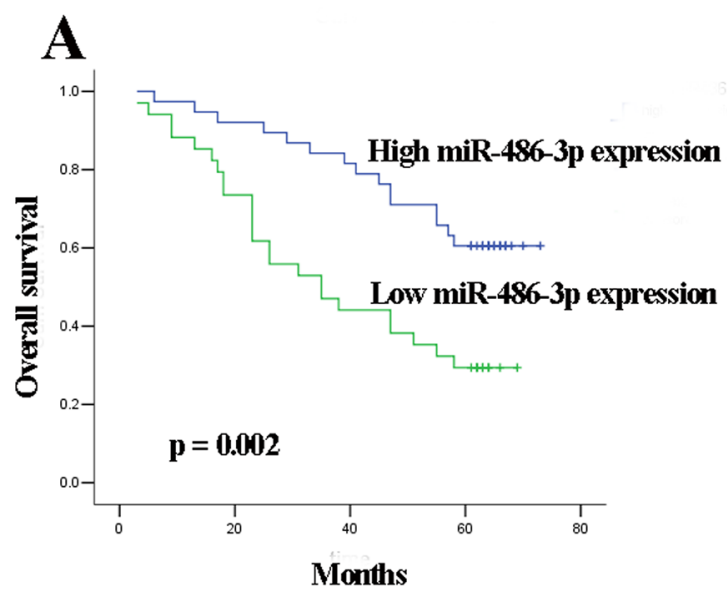

the predictive miRNA panel could perform efficiently in recurrent cases. Therefore, our research should be deemed as an important first step but not the definitive answer for clinical utility of these putative biomarkers.

Among four miRNAs revealed in this study, some are involved in genesis and development of cancer. miR486 has been shown to participant in general tumorigenesis by targeting the anti-apoptotic OLFM4 [30], SIRT1 [31], PIM-1 [32] and protumorigenic ARHGAP5 [33] in various types of cancer. Salah et al. proposed the miR-27a/miR$27 \mathrm{a}^{*}$ pair as a potential diagnostic and therapeutic target in managing osteosarcoma metastases [34]. In addition, MTA1 knockdown could significantly inhabit the expression of miR-103, which might prohibit the invasive phenotype of lung cancer [35]. At tissue level, miR-103-1

Figure 4: Kaplan-Meier curves for OS according to serum levels of A. miR-486-3p, B. miR-103a-3p in MIBC patients in validation set.

Table 3: Univariate and multivariate Cox proportional hazards regression model analysis of OS in MIBC patients in validation set

\begin{tabular}{|c|c|c|c|c|c|}
\hline \multirow[t]{2}{*}{ Parameters } & \multirow[t]{2}{*}{ Categories } & \multicolumn{2}{|c|}{$\begin{array}{c}\text { Univariate } \\
\text { analysis }\end{array}$} & \multicolumn{2}{|c|}{$\begin{array}{c}\text { Multivariate } \\
\text { analysis }\end{array}$} \\
\hline & & HR $(95 \%$ CI) & $p$ value & $\operatorname{HR}(95 \% \mathrm{CI})$ & $p$ value \\
\hline Age & $<65$ vs. $\geq 65$ & $1.505(0.789-2.872)$ & 0.215 & & \\
\hline Sex & Male vs. female & $0.670(0.262-1.715)$ & 0.404 & & \\
\hline Tumor stage & T2 vs. T3 vs. T4 & $1.830(1.201-2.788)$ & 0.005 & $1.682(1.053-2.687)$ & 0.030 \\
\hline Tumor grade & Low vs. high & $1.002(0.419-2.392)$ & 0.997 & & \\
\hline Lymph node metastasis & $\begin{array}{c}\text { Negative vs. } \\
\text { positive }\end{array}$ & $2.285(1.079-4.839)$ & 0.031 & $2.418(1.116-5.237)$ & 0.025 \\
\hline miR-422a-3p expression & Low vs. high & $1.083(0.578-2.030)$ & 0.804 & & \\
\hline miR-486-3p expression & Low vs. high & $0.380(0.199-0.728)$ & 0.004 & $0.500(0.257-0.974)$ & 0.042 \\
\hline miR-103a-3p expression & Low vs. high & $0.509(0.268-0.967)$ & 0.039 & $0.460(0.237-0.891)$ & 0.021 \\
\hline miR-27a-3p expression & Low vs. high & $1.660(0.881-3.130)$ & 0.117 & & \\
\hline
\end{tabular}


has been previously identified as up-regulated in $\mathrm{BC}$, indicating its potential involvement in the development and progression of $\mathrm{BC}$ [36]. Expression levels of miR422a-3p, miR-486-3p and miR-27a-3p, however, have not been reported in tissues of $\mathrm{BC}$. More researches on target genes of circulating miRNAs and complex mechanism that regulates the biogenesis of these four miRNAs in BC will be needed.

Currently, staging systems based on pathological grade and TNM stage are insufficient to predict clinical outcome. Different outcome for individuals with same tumor grade and stage calls for novel prognostic biomarkers and potential therapeutic targets $[37,38]$. Several studies demonstrated that miRNA expression significantly correlated with tumor aggressiveness and patient survival in MIBC tissues [39, 40]. However, prognostic values of serum miRNAs for MIBC have not been explored. In the present study, low miR-486$3 p$ and miR-103a-3p expression levels respectively correlated with shorter OS of MIBC. Taking a step further, Cox proportional hazards regression model analysis revealed that serum miR-486-3p and miR-103a-3p level were independent factors influencing OS of MIBC. Several investigators have reported similar findings in other types of cancer. Expression of miR-486 was found to be associated with cancer-specific mortality rate in non-small cell lung cancer [41]. Guo et al. demonstrated that miR-103/107 could serve as prognostic markers for OS of esophageal cancer [42]. Thus, we speculate that pretreatment serum levels of miR-486-3p and miR-103a$3 p$ might also serve as new prognostic biomarkers for MIBC.

Some limitations should be acknowledged. First, origin of circulating miRNAs was not fully understood. Some investigators suggested serum miRNA profiles was not simply a default product of circulating blood cells but might derive from tissues affected by diseases such as cancer [43]. More focus on release mechanisms of miRNAs in tumorigenesis and progression of BC may be a valuable avenue for increasing diagnostic specificity. Moreover, it is still unknown whether the miRNA panel is capable of discriminating MIBC from other types of invasive tumors. In addition, our study has limited generalizability. Thus, confirmation of our findings in a multicenter trial of larger independent samples is the objective of ongoing work.

In conclusion, we defined a distinctive serum miRNA signature for prediction of MIBC and identified independent prognostic variables for MIBC. These findings may provide a foundation for development of a novel noninvasive test to predict MIBC and determination of innovative therapeutic strategies.

\section{MATERIALS AND METHODS}

\section{Study design}

A multiphase and case-control study was designed to identify serum miRNAs as surrogate biomarkers for discriminating MIBC from NMIBC (Figure 5). In the discovery phase, serum from 6 MIBC patients, 6 NMIBC patients and 6 healthy donors were respectively subjected to Miseq sequencing and miRNAs with significant differences in expression levels among three groups were identified. In the training phase, candidate miRNAs were first tested by RT-qPCR in an independent cohort of serum samples from 40 MIBC patients, 40 NMIBC patients and 40 controls. Subsequently, miRNAs differentially expressed among the three groups were further tested in 71 MIBC patients, 71 NMIBC patients and 147 controls. These 111 MIBC patients and 111 NMIBC patients were used to construct the predictive miRNAs panel based on logistic regression model for MIBC. In the validation phase, serum from another independent cohort of 90 MIBC patients and 168 NMIBC patients were prospectively entered into the discriminatory model to validate the predictive accuracy of the constructed algorithm. Meanwhile, urine samples were obtained for traditional urine cytology. Additionally, MIBC patients were followed up at intervals of 3 months during the first 2 years and 6 months up to the fifth year, and the date of the latest record retrieved was March 31, 2015. This study was approved by the Clinical Research Ethics Committee of Qilu Hospital, Shandong University and informed consent was obtained from each participant.

\section{Patients and control subjects}

Patients with BC were recruited in the Department of Urology of Qilu Hospital, Shandong University between January 2005 and February 2010. Samples from BC patients were collected at the day before radical cystectomy and/or TUR. Tumor stage was determined according to the 2002 UICC TNM classification of $\mathrm{BC}$ and tumor grade was defined according to the WHO 2004 grading scheme. All MIBC patients were initially diagnosed with muscle-invasive diseases. Control participants without history of BC were recruited from a large pool of individuals seeking a routine health checkup at the Healthy Physical Examination Centre of Qilu Hospital, Shandong University. People who showed no evidence of disease were selected as tumor-free controls. The demographic and clinical features of participants are summarized in Supplementary Table S2.

\section{Serum preparation}

Venous blood samples were collected by vena puncture from each participant before any treatment. Serum was separated via centrifugation at $1,600 \mathrm{~g}$ for 10 min at $4^{\circ} \mathrm{C}$ within $2 \mathrm{hr}$ of collection, followed by a second centrifugation at $16,000 \mathrm{~g}$ for $10 \mathrm{~min}$ at $4^{\circ} \mathrm{C}$ to eliminate residual cells debris. Supernatant serum was then stored at $-80^{\circ} \mathrm{C}$ till use. 


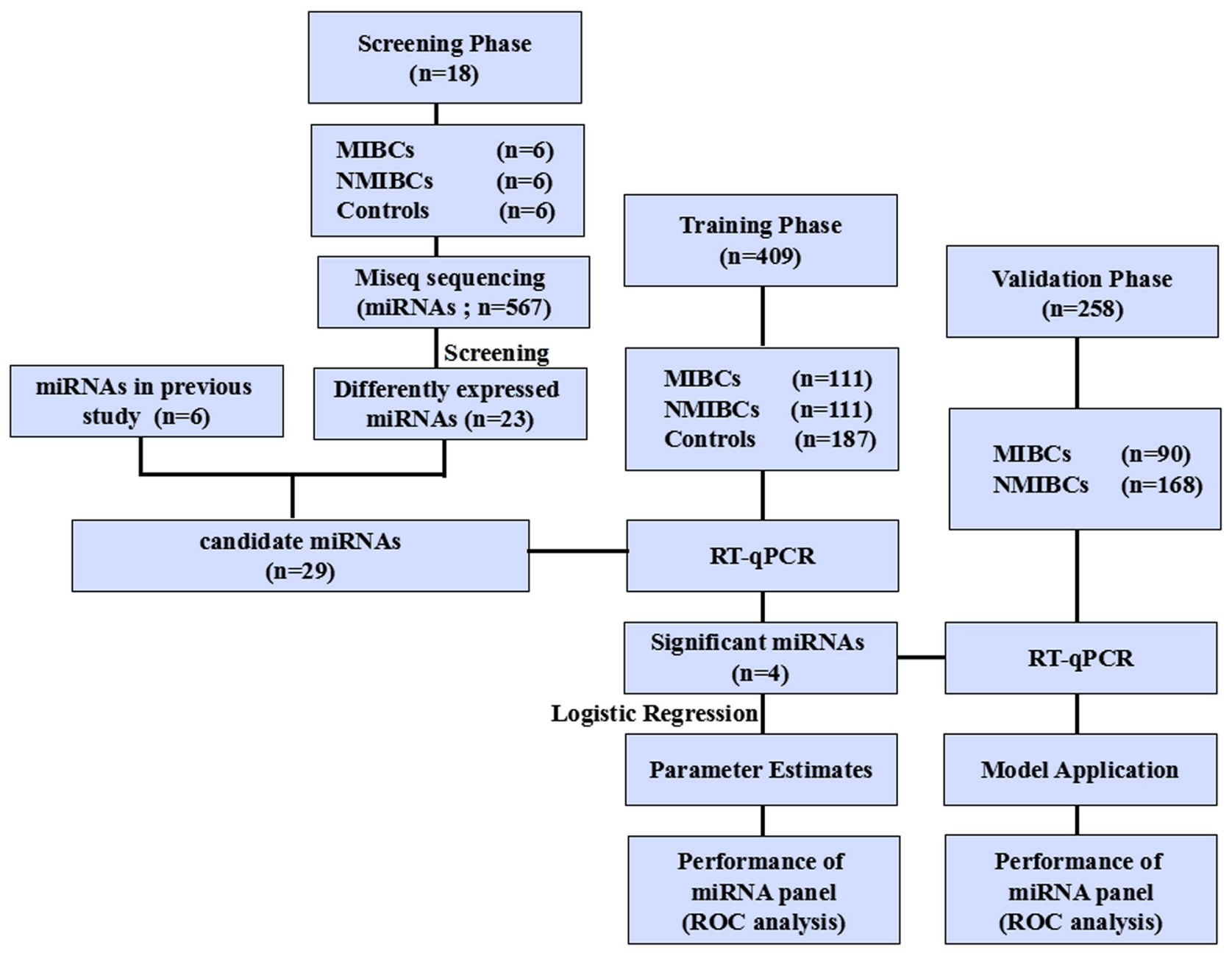

Figure 5: Study outline.

\section{Miseq sequencing}

For Miseq sequencing, total RNA was extracted and purified using miRNeasy Mini Kit (Qiagen, Valencia, CA, USA) following the protocol provided by the manufacturer. Briefly, a pair of adaptors was ligated sequentially to the 3 ' and 5 ' ends of miRNA, and the ligated miRNA molecules were amplified by RT-qPCR to construct a cDNA library. Quality of the library was measured by the KAPA RT-qPCR kit and cDNAs with concentrations of higher than $1 \mathrm{nM}$ and no dimmer contamination were used directly for sequencing analysis (Miseq sequencer, Illumina, San Diego, CA, USA). The final reads of miRNA were identified by normalization with the total reads of all called miRNAs in the sample. Bioinformatics analysis was conducted by searching against the miRBase 17.0 to determine known mature miRNAs.

\section{Quantification of miRNAs by RT-qPCR analysis}

The $2 \times$ preparation buffer that consisted of $2.5 \%$ Tween 20 (EMD Chemicals, Gibbstown, NJ), $50 \mathrm{mmol} / \mathrm{L}$ Tris (Sigma-Aldrich, St.Louis, MO), and 1mmol/L EDTA
(Sigma-Aldrich, St.Louis, MO) was prepared [44]. First, $3 \mu \mathrm{l}$ of serum were mixed with $3 \mu \mathrm{l}$ of $2 \times$ preparation buffer. Then, the $6 \mu l$ mixture were reverse transcribed in a $20-\mu 1$ reverse transcription (RT) reaction volume system including $10 \mu \mathrm{l}$ of $2 \times$ miRNA Reaction Buffer Mix, $2 \mu \mathrm{l}$ of miRNA Primescript RT Enzyme Mix and $2 \mu \mathrm{l}$ of $0.1 \%$ BSA. The reactions were incubated at $37^{\circ} \mathrm{C}$ for $60 \mathrm{~min}$, followed by $85^{\circ} \mathrm{C}$ for $5 \mathrm{~s}$ and $4^{\circ} \mathrm{C}$ for $60 \mathrm{~min}$. The generated cDNAs were centrifuged at $16,000 \mathrm{~g}$ for $10 \mathrm{~min}$ at $4^{\circ} \mathrm{C}$. The 25- $\mu$ l RT-qPCR reaction system contained $12.5 \mu \mathrm{l}$ of SYBR Premix Ex Taq II, $0.5 \mu$ of Dye II, $2 \mu 1$ of $5 \mu \mathrm{M}$ of forward primer, $1 \mu \mathrm{l}$ of $10 \mu \mathrm{M}$ of Uni-miR RT-qPCR Primer, $7 \mu \mathrm{l}$ of $\mathrm{ddH}_{2} \mathrm{O}$ and $2 \mu \mathrm{l}$ of template cDNA. The reactions were incubated at $95^{\circ} \mathrm{C}$ for $30 \mathrm{~s}$, followed by 45 cycles of $95^{\circ} \mathrm{C}$ for $5 \mathrm{~s}$ and $57^{\circ} \mathrm{C}$ for $34 \mathrm{~s}$. All reactions were performed in triplicate in ABI PRISM 7500 Sequence Detection System (Applied Biosystems, Foster City, CA) using the SYBR PrimeScript miRNA RT-qPCR Kit (Takara Bio Inc). Specificity of the RT-qPCR product was confirmed with melting curve analysis and $\mathrm{Ct}$ values were determined using default threshold setting. miRNAs with a Ct value of more than 35 and a detection rate of less than 
$75 \%$ in any group were excluded from further analyses. The combination of miR-16-5p and miR-193a-5p were used as reference genes [45]. The relative expression fold change was calculated by using the $2^{-\Delta \Delta \mathrm{Ct}}$ method [46].

\section{Urine cytology determination}

Midstream urine sediments were separated via centrifugation at $1,300 \mathrm{~g}$ for $10 \mathrm{~min}$ and immediately processed for cytological examinations by two cytopathologists in a blinded fashion. The cytology was categorized as positive if cancer cells or cells with atypical changes indicating malignancy were found, and negative if mild to moderate atypical changes were observed.

\section{Statistical analysis}

Kolmogorov-Smirnov test was used to determine the normality of the distribution of data in each group. Data were presented as median (interquartile range). Difference of miRNA levels among multiple groups in Miseq sequencing was determined by Bonferroni adjustment method. Mann-Whitney U tests were employed to compare differences of miRNAs between two groups in further validation. Receiver operating characteristic (ROC) curves were established to discriminate MIBC from NMIBC. Area under the ROC curve (AUC) was used as an accuracy index for evaluating the predictive performance of constructed miRNA panel [47]. The Youden index (sensitivity + specificity -1 ) was used to determine the optimal cutoff point [48]. Survival curves were estimated with Kaplan-Meier method and comparisons were conducted using log-rank test. Cox proportional hazards regression model was employed to identify independent prognostic factors. Matlab software (Matlab, R2013a) was employed for logistic regression analysis, MedCalc 9.3.9.0 (MedCalc, Mariakerke, Belgium) was used for ROC analysis, and other analysis was performed by SPSS version 17.0 software (SPSS Inc., Chicago, IL). Statistical significance was defined as two-sided $p$ value $<0.05$.

\section{ACKNOWLEDGMENTS}

This work was supported by National Natural Science Foundation of China (No. 81271916, 81472025 and 81501822), Natural Science Foundation of Shandong (No. ZR2014HP001), Shandong Technological Development Project (STDP, 2014GSF118016 and 2015GSF118052), Science Foundation of Qilu Hospital of Shandong University, and the Fundamental Research Funds of Shandong University.The authors thank Dr. Chengjun Zhou (Department of Pathology, Second Hospital of Shandong University) and Dr. Junhui Zhen (Department of Pathology, Qilu Hospital, Shandong University) for their assistance in evaluation of cytology and histology.

\section{CONFLICTS OF INTEREST}

The authors declare that no potential conflicts of interest were disclosed.

\section{REFERENCES}

1. Siegel RL, Miller KD, Jemal A. Cancer statistics, 2015. CA Cancer J Clin. 2015;65:5-29.

2. Kaufman DS, Shipley WU, Feldman AS. Bladder cancer. Lancet. 2009;374:239-249.

3. Brausi MA. Primary prevention and early detection of bladder cancer: two main goals for urologists. Eur Urol. 2013;63:242-243.

4. Hollenbeck BK, Miller DC, Taub D, Dunn RL, Khuri SF, Henderson WG, Montie JE, Underwood W 3rd, Wei JT. Risk factors for adverse outcomes after transurethral resection of bladder tumors. Cancer. 2006; 106:1527-1535.

5. Schiffer E, Vlahou A, Petrolekas A, Stravodimos K, Tauber R, Geschwend JE, Neuhaus J, Stolzenburg JU, Conaway MR, Mischak H, Theodorescu D. Prediction of muscleinvasive bladder cancer using urinary proteomics. Clin Cancer Res. 2009;15:4935-4943.

6. Ambros V. The functions of animal microRNAs. Nature. 2004; 431:350-355.

7. Bartel DP. MicroRNAs: genomics, biogenesis, mechanism, and function. Cell. 2004;116:281-297.

8. Yang Y, Li F, Saha MN, Abdi J, Qiu L, Chang H. miR137 and miR-197 Induce Apoptosis and Suppress Tumorigenicity by Targeting MCL-1 in Multiple Myeloma. Clin Cancer Res. 2015;21:2399-2411.

9. Wong CM, Wei L, Au SL, Fan DN, Zhou Y, Tsang FH, Law CT, Lee JM, He X, Shi J, Wong CC, Ng IO. MiR200b/200c/429 subfamily negatively regulates Rho/ROCK signaling pathway to suppress hepatocellular carcinoma metastasis. Oncotarget. 2015;6:13658-13670. doi: 10.18632/ oncotarget. 3700 .

10. Liu DS, Read M, Cullinane C, Azar WJ, Fennell CM, Montgomery KG, Haupt S, Haupt Y, Wiman KG, Duong CP, Clemons NJ, Phillips WA. APR-246 potently inhibits tumour growth and overcomes chemoresistance in preclinical models of oesophageal adenocarcinoma. Gut. 2015;64:1506-1516.

11. Huang TT, Ping YH, Wang AM, Ke CC, Fang WL, Huang $\mathrm{KH}$, Lee HC, Chi CW, Yeh TS. The reciprocal regulation loop of Notch2 pathway and miR-23b in controlling gastric carcinogenesis. Oncotarget. 2015;6:18012-18026. doi: 10.18632/oncotarget.4000.

12. Zhang $C$, Wang $C$, Chen $X$, Yang C, Li K, Wang J, Dai J, Hu Z, Zhou X, Chen L, Zhang Y, Li Y, Qiu H, et al. Expression profile of microRNAs in serum: a fingerprint for esophageal squamous cell carcinoma. Clin Chem. 2010;56:1871-1879. 
13. Yamada A, Horimatsu T, Okugawa Y, Nishida N, Honjo H, Ida H, Kou T, Kusaka T, Sasaki Y, Yagi M, Higurashi T, Yukawa N, Amanuma Y, et al. Serum miR-21, miR-29a, and miR-125b Are Promising Biomarkers for the Early Detection of Colorectal Neoplasia. Clin Cancer Res. 2015; 21:4234-42.

14. Chen X, Hu Z, Wang W, Ba Y, Ma L, Zhang C, Wang C, Ren Z, Zhao Y, Wu S, Zhuang R, Zhang Y, Hu H, et al. Identification of ten serum microRNAs from a genome-wide serum microRNA expression profile as novel noninvasive biomarkers for nonsmall cell lung cancer diagnosis. Int $\mathrm{J}$ Cancer. 2012;130:1620-1628.

15. Liu R, Zhang C, Hu Z, Li G, Wang C, Yang C, Huang D, Chen X, Zhang H, Zhuang R, Deng T, Liu H, Yin J, et al. A five-microRNA signature identified from genomewide serum microRNA expression profiling serves as a fingerprint for gastric cancer diagnosis. Eur J Cancer. 2011;47:784-791.

16. Li J, Liu Y, Wang C, Deng T, Liang H, Wang Y, Huang D, Fan Q, Wang X, Ning T, Liu R, Zhang CY, Zen K, et al. Serum miRNA expression profile as a prognostic biomarker of stage II/III colorectal adenocarcinoma. Sci rep. 2015;5:12921.

17. Kleivi Sahlberg K, Bottai G, Naume B, Burwinkel B, Calin GA, Borresen-Dale AL, Santarpia L. A serum microRNA signature predicts tumor relapse and survival in triple-negative breast cancer patients. Clin Cancer Res. 2015;21:1207-1214.

18. Jiang X, Du L, Wang L, Li J, Liu Y, Zheng G, Qu A, Zhang X, Pan H, Yang Y, Wang C, et al. Serum microRNA expression signatures identified from genome-wide microRNA profiling serve as novel noninvasive biomarkers for diagnosis and recurrence of bladder cancer. Int J Cancer. 2015; $136: 854-862$

19. Clark PE, Agarwal N, Biagioli MC, Eisenberger MA, Greenberg RE, Herr HW, Inman BA, Kuban DA, Kuzel TM, Lele SM, Michalski J, Pagliaro LC, Pal SK, et al. Bladder cancer. J Natl Compr Canc Netw. 2013;11:446-475.

20. Catto JW, Miah S, Owen HC, Bryant H, Myers K, Dudziec E, Larre S, Milo M, Rehman I, Rosario DJ, Di Martino E, Knowles MA, Meuth M, et al. Distinct microRNA alterations characterize high- and low-grade bladder cancer. Cancer Res. 2009;69:8472-8481.

21. Wiklund ED, Bramsen JB, Hulf T, Dyrskjot L, Ramanathan R, Hansen TB, Villadsen SB, Gao S, Ostenfeld MS, Borre M, Peter ME, Ørntoft TF, Kjems J, et al. Coordinated epigenetic repression of the miR-200 family and miR-205 in invasive bladder cancer. Int J Cancer. 2011;128:1327-1334.

22. Segersten U, Spector Y, Goren Y, Tabak S, Malmstrom PU. The role of microRNA profiling in prognosticating progression in Ta and T1 urinary bladder cancer. Urol Oncol. 2014;32:613-618.

23. Sternberg CN, Donat SM, Bellmunt J, Millikan RE, Stadler W, De Mulder P, Sherif A, von der Maase H, Tsukamoto T, Soloway MS. Chemotherapy for bladder cancer: treatment guidelines for neoadjuvant chemotherapy, bladder preservation, adjuvant chemotherapy, and metastatic cancer. Urology. 2007;69:62-79.

24. Carrion R, Seigne J. Surgical management of bladder carcinoma. Cancer Control. 2002;9:284-292.

25. Kriebel S, Schmidt D, Holdenrieder S, Goltz D, Kristiansen G, Moritz R, Fisang C, Muller SC, Ellinger J. Analysis of tissue and serum microRNA expression in patients with upper urinary tract urothelial cancer. PloS one. 2015;10:e0117284.

26. Du M, Shi D, Yuan L, Li P, Chu H, Qin C, Yin C, Zhang Z, Wang M. Circulating miR-497 and miR-663b in plasma are potential novel biomarkers for bladder cancer. Sci Rep. 2015;5:10437.

27. Adam L, Wszolek MF, Liu CG, Jing W, Diao L, Zien A, Zhang JD, Jackson D, Dinney CP. Plasma microRNA profiles for bladder cancer detection. Urol Oncol. 2013;31:1701-1708.

28. Feng Y, Liu J, Kang Y, He Y, Liang B, Yang P, Yu Z. miR19a acts as an oncogenic microRNA and is up-regulated in bladder cancer. J Exp Clin Cancer Res. 2014;33:67.

29. Feng Y, Kang Y, He Y, Liu J, Liang B, Yang P, Yu Z. microRNA-99a acts as a tumor suppressor and is downregulated in bladder cancer. BMC urology. 2014;14:50.

30. Oh HK, Tan AL, Das K, Ooi CH, Deng NT, Tan IB, Beillard E, Lee J, Ramnarayanan K, Rha SY, Palanisamy N, Voorhoeve PM, Tan P, et al. Genomic loss of miR486 regulates tumor progression and the OLFM4 antiapoptotic factor in gastric cancer. Clin Cancer Res. 2011;17:2657-2667.

31. Kim YJ, Hwang SH, Lee SY, Shin KK, Cho HH, Bae YC, Jung JS. miR-486-5p induces replicative senescence of human adipose tissue-derived mesenchymal stem cells and its expression is controlled by high glucose. Stem Cells Dev. 2012;21:1749-1760.

32. Pang W, Tian X, Bai F, Han R, Wang J, Shen H, Zhang X, Liu Y, Yan X, Jiang F, Xing L, et al. Pim-1 kinase is a target of miR-486-5p and eukaryotic translation initiation factor $4 \mathrm{E}$, and plays a critical role in lung cancer. Mol Cancer. 2014;13:240.

33. Wang J, Tian X, Han R, Zhang X, Wang X, Shen H, Xue L, Liu Y, Yan X, Shen J, Mannoor K, Deepak J, Donahue JM, et al. Downregulation of miR-486-5p contributes to tumor progression and metastasis by targeting protumorigenic ARHGAP5 in lung cancer. Oncogene. 2014;33:1181-1189.

34. Salah Z, Arafeh R, Maximov V, Galasso M, Khawaled $\mathrm{S}$, Abou-Sharieha S, Volinia S, Jones KB, Croce CM, Aqeilan RI. miR-27a and miR-27a* contribute to metastatic properties of osteosarcoma cells. Oncotarget. 2015;6:49204935. doi: 10.18632/oncotarget.3025.

35. Zhu X, Zhang X, Wang H, Song Q, Zhang G, Yang L, Geng J, Li X, Yuan Y, Chen L. MTA1 gene silencing inhibits invasion and alters the microRNA expression profile of human lung cancer cells. Oncol Rep. 2012;28:218-224. 
36. Gottardo F, Liu CG, Ferracin M, Calin GA, Fassan M, Bassi P, Sevignani C, Byrne D, Negrini M, Pagano F, Gomella LG, Croce CM, Baffa R. Micro-RNA profiling in kidney and bladder cancers. Urol Oncol. 2007;25:387-392.

37. Chopin D, Cappellen D, Fradvanyi F, Gattegno B. Fundamental bases of urothelial carcinogenesis [Article in French]. Prog Urol. 2001;11:879-924.

38. Lee R, Droller MJ. The natural history of bladder cancer. Implications for therapy. Urol Clin N Am. 2000;27:1-13.

39. Pignot G, Cizeron-Clairac G, Vacher S, Susini A, Tozlu S, Vieillefond A, Zerbib M, Lidereau R, Debre B, AmsellemOuazana D, Bieche I. microRNA expression profile in a large series of bladder tumors: identification of a 3-miRNA signature associated with aggressiveness of muscle-invasive bladder cancer. Int J Cancer. 2013;132:2479-2491.

40. Rosenberg E, Baniel J, Spector Y, Faerman A, Meiri E, Aharonov R, Margel D, Goren Y, Nativ O. Predicting progression of bladder urothelial carcinoma using microRNA expression. BJU Int. 2013;112:1027-1034.

41. Hu Z, Chen X, Zhao Y, Tian T, Jin G, Shu Y, Chen Y, Xu L, Zen K, Zhang C, Shen H. Serum microRNA signatures identified in a genome-wide serum microRNA expression profiling predict survival of non-small-cell lung cancer. J Clin Oncol. 2010;28:1721-1726.

42. Guo Y, Chen Z, Zhang L, Zhou F, Shi S, Feng X, Li B, Meng X, Ma X, Luo M, Shao K, Li N, Qiu B.
Distinctive microRNA profiles relating to patient survival in esophageal squamous cell carcinoma. Cancer Res. 2008;68:26-33.

43. Chen X, Ba Y, Ma L, Cai X, Yin Y, Wang K, Guo J, Zhang Y, Chen J, Guo X, Li Q, Li X, Wang W. Characterization of microRNAs in serum: a novel class of biomarkers for diagnosis of cancer and other diseases. Cell Res. 2008; 18:997-1006.

44. Asaga S, Kuo C, Nguyen T, Terpenning M, Giuliano AE, Hoon DS. Direct serum assay for microRNA-21 concentrations in early and advanced breast cancer. Clin Chem. 2011;57:84-91.

45. Wang L, Liu Y, Du L, Li J, Jiang X, Zheng G, Qu A, Wang H, Wang L, Zhang X, Liu H, Pan H, Yang Y, et al. Identification and validation of reference genes for the detection of serum microRNAs by reverse transcriptionquantitative polymerase chain reaction in patients with bladder cancer. Mol Med Rep. 2015;12:615-622.

46. Schmittgen TD, Lee EJ, Jiang J, Sarkar A, Yang L, Elton TS, Chen C. Real-time PCR quantification of precursor and mature microRNA. Methods. 2008;44:31-38.

47. Hanley JA, McNeil BJ. A method of comparing the areas under receiver operating characteristic curves derived from the same cases. Radiology. 1983;148:839-843.

48. Youden WJ. Index for rating diagnostic tests. Cancer. 1950;3:32-35. 